\title{
Entorno a la problemática del recurso agua ante los megaproyectos de transporte. Estudio de caso Zona Metropolitana de la Ciudad de Querétaro
}

\author{
Rebeca Granados-Ramírez ${ }^{1, *}$, Alma Villaseñor-Franco ${ }^{2, * *}$ \\ ${ }^{1}$ Departamento de Geografía Física, Instituto de Geografía, Universidad Nacional Autónoma de México, \\ México, D.F. C. P. 04510, México. \\ ${ }^{2}$ Unidad Académica de Ciencias de la Tierra. Universidad Autónoma de Guerrero, \\ Domicilio conocido, Taxco, Guerrero. \\ rrebeca@igg.unam.mx \\ **alvif27@yahoo.com.mx
}

\section{Resumen}

El agua es considerada como un recurso renovable. Sin embargo, la sobreexplotación (abastecimiento a ciudades, tanto para consumo humano, industrial y agrícola), así como la contaminación, dan como resultado una gran demanda del mismo, tornándose en un bien estratégico.

En el actual proceso de globalización se impulsan diversos programas de inversión, tales como los megaproyectos de transporte, mismos que a su vez impulsan la urbanización, desarrollo de corredores viales e industriales; impactando en el medio natural e incrementándose la demanda del recurso agua, entre otros.

La ciudad de Querétaro posee una ubicación estratégica donde convergen importantes vías de comunicación, generándose un considerable tráfico de pasajeros y carga, del norte, occidente y sur del territorio. Uno de los proyectos que impactarán en ésta ciudad es el proyecto denominado "Gran Visión”. La realización de estos megaproyectos de transporte, modifica el curso tendencial de crecimiento de las ciudades; incrementándose la demanda de recursos, entre otros el agua. Uno de los retos más complejos que enfrenta el país y las entidades federativas es la urgente necesidad de adoptar esquemas de aprovechamiento sustentable del vital líquido.

Ante esta panorámica el objetivo de esta investigación es resaltar la función que posee la ciudad de Querétaro ante actuales megaproyectos de transporte, importancia de la vinculación del transporte con la urbanización y a su vez con el impacto en los recursos. Se desarrolla el panorama actual de los megaproyectos en el que está inmersa la ciudad de Querétaro, el crecimiento histórico, actual y tendencial de la mancha urbana y la demanda futura del recurso agua, consecuencia de la aplicación de estos proyectos.

Palabras clave: recurso renovable, Querétaro, ubicación estratégica, demanda, recurso agua.

\begin{abstract}
Water is considered a renewable resource. Nevertheless, its overexploitation (when supplied to cities, for human, industrial and agricultural consumption), as well as its pollution, result in a high demand for this product, thus becoming a strategic resource.

In the process of globalization, diverse investment programs are created, such as big transportation projects, which lead to further urbanization and the construction of new roads and industrial areas. This impacts the environment by increasing the demand for water, and in other different ways as well.

The City of Queretaro is a strategic location where important routes of communication converge. This generates a considerable amount of traffic of passengers and freight, from the north, west and south of the territory. One of the projects that will strongly hit
\end{abstract}


this city is named "Great Vision". These big transportation projects typically modify cities' normal course of growth, thus increasing the demand for natural resources, including water. One of the most complex challenges that Mexico and its federal entities face is the urgent necessity to adopt schemes of sustainable exploitation of this highly-valued resource.

Under this perspective, the main goal of this paper is to emphasize the role that the City of Queretaro plays in current big transportation projects, in the importance of coordinating transportation works with urbanization development and, in turn, with the impact in natural resources. In addition, it describes the current situation where big projects have been undertaken by the City of Queretaro, the current and historical growth of urban areas, and the future demand for water, a direct consequence of the implementation of these projects.

Key words: renewable resource, Queretaro, strategic location, demand, resource water.

\section{Introducción}

Actualmente, los procesos de la globalización están generando una nueva configuración territorial a nivel mundial, con importantes cambios en la jerarquía y funcionalidad de las zonas urbanas y metropolitanas (Ampe, 1995).

Las grandes obras de infraestructura, como los aeropuertos, redes carreteras, etc.; traen consigo masivas urbanizaciones formales e informales (Aguilar, 2006). En México la implementación de tratados comerciales, ha conformado rutas (aéreas y carreteras), entre otros: Tratado de Libre Comercio de Norteamérica (TLCAN), la ruta del Plan Puebla Panamá (PPP) (SCT, 2001a). En dichas rutas se han implementado una serie de megaproyectos, ente otros, la conformación de ejes carreteros, mismos que ha ocasionado que aquellos lugares por donde pasan, impulsen su desarrollo y se incremente el proceso de urbanización (Banister, 1995).

La evolución de los espacios metropolitanos, dinámica de organización funcional, accesibilidad, estructura económica y espacial; ha estado vinculada con la implementación de megaproyectos de transporte (MPT).

Los MPT ocasionan grandes impactos (territoriales, económicos-sociales y ambientales). Por ejemplo el crecimiento espacial acelerado de algunas ciudades hacia su periferia, resultado de las anexiones de municipios que la rodean. El ritmo y las modalidades de esta extensión espacial varía según el dinamismo de las ciudades (Hanson, 1986).

Así, la infraestructura carretera, ha generado grandes cambios en el uso del suelo y estructura de las ciudades. De aquí se destaca la importancia del estudio de los MPT en ciudades que se ven implicadas, se precisa considerar que estos proyectos alteran las tendencias de crecimiento y su consecuente demanda de servicios, entre ellos el agua.

Entre los impactos de los MPT en una región son: económicos que posibilitan el desarrollo de proyectos de inversión (industriales, turísticos, etc.), territoriales (vinculados al desarrollo urbano) y ambientales debido a que al implementar la construcción de un MPT va a tener efectos negativos en el medio físico.

\section{Zona de estudio}

El municipio de Querétaro se localiza entre las coordenadas $20^{\circ} 30^{\prime}$ a $20^{\circ} 54^{\prime}$ de latitud Norte y a los $100^{\circ} 17^{\prime}$ a $100^{\circ} 36^{\prime}$ de longitud Oeste. Colinda con los municipios: El Marqués, Huimilpan y Corregidora; dicha aglomeración urbana continua y se le ha denomina Zona Metropolitana de la Ciudad de Querétaro (ZMCQ).

La altura media sobre el nivel del mar varía de 1900 a 2 $460 \mathrm{msnm}$. En la llanura del municipio se desarrolla la ciudad de Querétaro y se extiende hasta a las colonias satélites del Noreste y Suroeste como son la Zona Industrial, Obrera, Cerrito Colorado, San Pedro Mártir, La Colmena, Carrillo Puerto, Santa María Magdalena, Los Cobos, Santa María Venegas, El Retablo y Club Campestre (INEGI, 2002).

\section{La infraestructura carretera}

El municipio de Querétaro tiene 107.7 Km. de carretera federal y $137.5 \mathrm{Km}$. de camino rural asfaltado y es uno de los principales centros de comunicación del país. Las vías más importantes son: Av. 5 de Febrero (carretera México - Querétaro - Piedras Negras); Panamericana (México Querétaro - Cd. Juárez), carretera federal No. 90 (carretera México - Querétaro - Guadalajara - Tijuana) y la carretera federal No. 120 (Querétaro - Jalpan - Tampico), así como una importante red carretera en buen estado que comunica con los municipios aledaños.

La infraestructura carretera en el municipio ha crecido significativamente, al igual que la urbanización, actualmente existe conurbación con el municipio de Corregidora, El Marqués y Huimilpan, dicha aglomeración urbana continua y se le ha denomina Zona Metropolitana de la Ciudad de Querétaro (ZMCQ).

\section{Los megaproyectos de transporte y su participación en el crecimiento urbano}

Los MPT se define como aquel proyecto carretero que, por su magnitud y complejidad, requiere de fuertes 
inversiones, además de ocasionar grandes impactos territoriales, económicos, sociales y ambientales (Villaseñor, 2004). Posibilita el desarrollo y evolución de las redes de conexión urbana e interurbana y crecimiento acelerado de la periferia, resultado de las anexiones de municipios cercanos. Figura 1.

El estado y la ciudad de Querétaro poseen una ubicación geográfica estratégica y aunado al proceso de globalización ha favorecido a la construcción de distintos obras. En la década de los noventas se inició la firma de acuerdos y tratados comerciales como el Tratado de Libre Comercio (TLC) con Estados Unidos y Canadá, con lo que nuestro país ingreso a nuevos mercados e inversiones y propició el aumento a las exportaciones principalmente hacia los Estados Unidos. Como consecuencia de lo anterior se construyo la carretera libre de cuatro carriles México- Querétaro:

La modernización de las carreteras que confluyen y cruzan a la ZMCQ, México-Guadalajara y México-Nuevo
Laredo (esta última llamada del TLC), favoreció su consolidación como un centro nodal y dio un fuerte impulso a la actividad industrial y comercial especialmente relacionado con los Estados Unidos, particularmente a lo largo del eje de comunicación.

La ZMCQ al localizarse en un excelente punto de accesibilidad hacia la zona norte, sur y occidente del país, se ve favorecida por la confluencia de rutas y que además al vincularse con los MPT que se encuentran en desarrollo, tendrá como consecuencia que se impulse aún más el crecimiento urbano. La metrópoli se ha ido expandiendo siguiendo los ejes de comunicación e incorporando territorios y a la vez, la conurbación de municipios cercanos al de Querétaro en lugares alejados del núcleo metropolitano.

El plan de desarrollo reconoce la prioridad de fortalecer un sistema de ciudades así como la existencia de una fuerte dinámica a lo largo de los ejes carreteros. La ZMCQ cuenta con una ubicación privilegiada como punto de convergencia

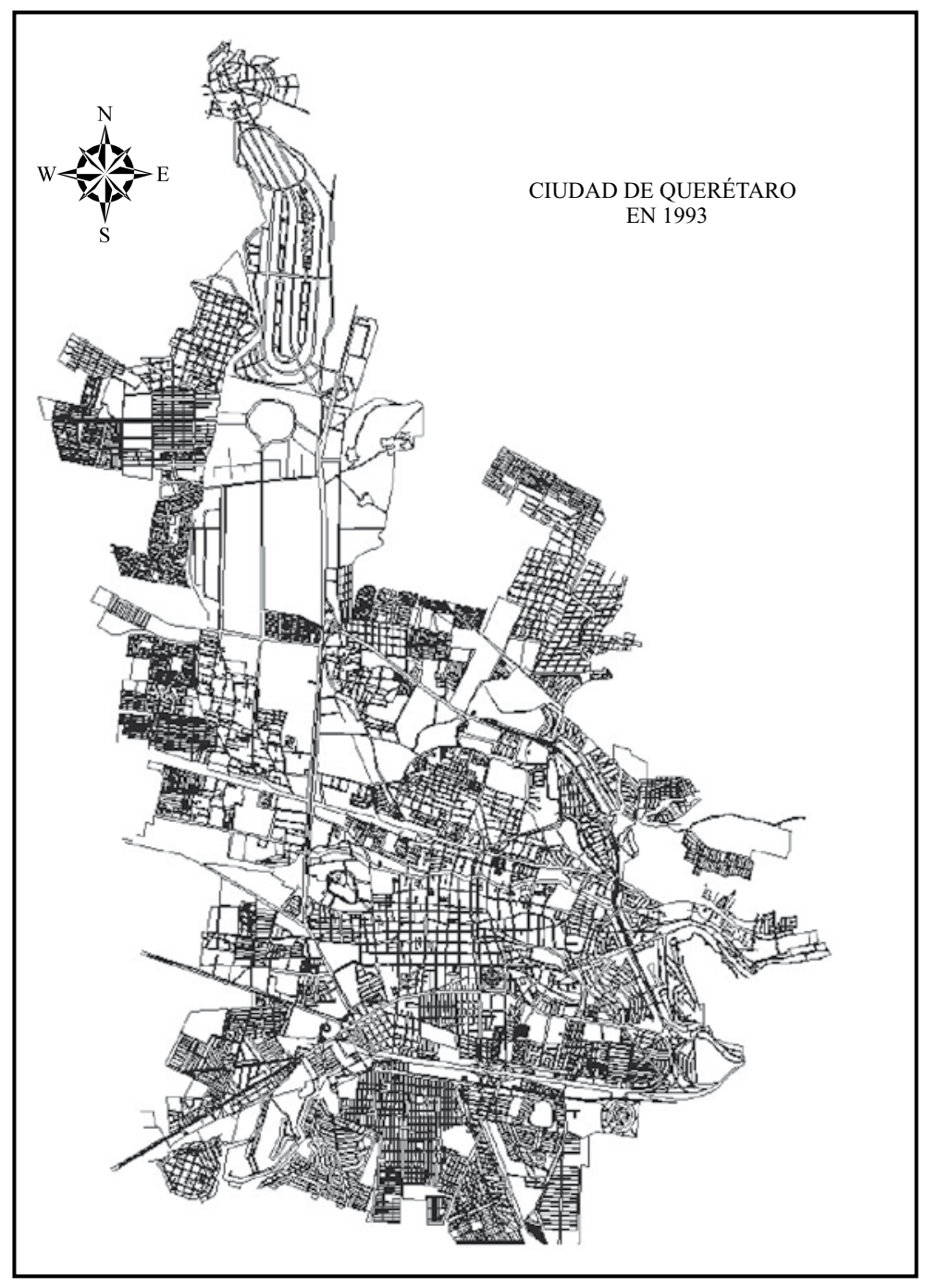

Figura 1. Ciudad de Querétaro, traza urbana 1993. 
de las principales vías de comunicación a nivel nacional, como son las carretera federal no. 57 y la carretera federal no. 45 que enlazan a la Zona Metropolitana de la Ciudad de México con el Centro, Occidente y Norte del país.

Esta entidad presenta dinámica creciente en cuanto a población que se ve atraída a las grandes concentraciones urbanas, modificando su forma de vida, producción y reproducción. El crecimiento natural y movimientos de población en la ZMCQ provocan el crecimiento acelerado de la población, con su correspondiente presión por necesidades de suelo, fuentes de empleo, servicios básicos, equipamiento urbano y vivienda.

\section{MPT "Gran Visión"}

El objetivo de este proyecto carretero es aprovechar el potencial productivo de las diversas localidades y polos de desarrollo. Se centra en la integración del Corredor del Altiplano, que une a la región Centro del País con el Golfo de México, así como en la conexión, a través de la construcción de diversos ramales carreteros, de las microrregiones de los estados que forman parte del proyecto, con los corredores carreteros que cruzan por esta región (SCT, 2001b); http://www.sct.gob.mx, por lo que su construcción incrementará los niveles de conectividad y accesibilidad de la ZMCQ.

Cabe señalar que actualmente la línea estratégica del Plan de Desarrollo de Querétaro se encuentra enfocada hacia un desarrollo integral sustentable, con una adecuada planeación urbana y que además considera importante el conducir dicha planeación en función de las nuevas dinámicas espaciales, en donde se deben incluir estos MPT o los futuros proyectos. Si bien el estado cuenta con esta herramienta de planeación, es importante considerar las tendencias de crecimiento de las zonas urbanas, las cuales se darán a lo largo de los principales corredores viales.

Aunque en la región centro del país se registran avances importantes en infraestructura que han impulsado su crecimiento, ese mismo dinamismo demanda ahora la construcción de nuevas obras para descongestionar y hacer más eficientes sus intercambios inter e intrarregionales y, de esa manera, elevar su competitividad. Para esta región es imperiosa la necesidad de contar con enlaces más eficientes con el Golfo de México y otras regiones, como la Centro Occidente y, a través de ella, con las Noroeste y Noreste (H. Ayuntamiento de Querétaro, 1997).

En general, todas las regiones requieren consolidar su integración a través de sistemas de comunicaciones y transportes eficientes y seguros, de aquí la importancia de estos corredores en esta gran región Figura 2.

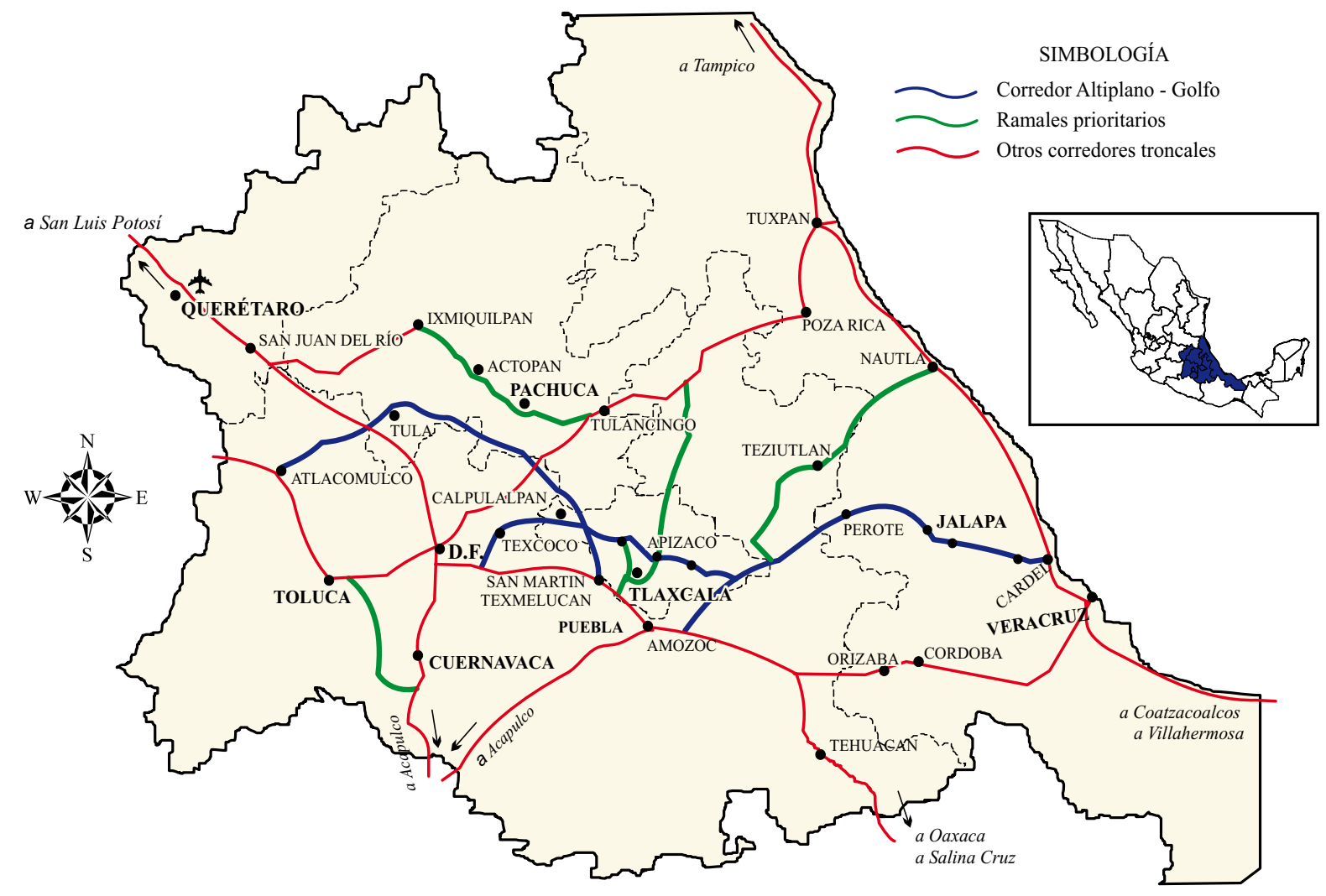

Figura 2. Proyecto regional “Gran Vísión”. Fuente SCT 2004. 


\section{Crecimiento urbano}

El crecimiento urbano es uno de los grandes problemas actuales del mundo en generales y de México en particular. En los últimos 30 años el crecimiento demográfico ha aumentado: en zonas rurales, se incrementa lentamente, mientras que en el medio urbano se incrementa rápidamente. En general de 1970 a 2000 se registró un cambio notable al ocupar la población urbana el $67 \%$ en relación a la total. Esto indica que hay crecimiento natural en estas zonas aunado a un continuo movimiento de población con dirección a la vida urbana (Gutiérrez- McGregor y González, 2004).

En 30 años la población rural aumentó 1.3 veces, con un incremento medio anual de $0.9 \%$; mientas la urbana 3.4 $\%$, (crecimiento total del país $2.3 \%$ ) aumentó su valor 2.7 veces. En 1970 vivían en áreas urbanas 23.7 millones de habitantes, a fines del 2000 fueron 65.3 millones Figura 3.

(Gutiérrez- McGregor y González, 2004) Marcan cuatro hechos que dan lugar al aumento de población urbana, dentro del los cuales se encuentra el crecimiento natural y éxodo de la población que habita en zonas rurales, mismas que se asientas en zonas adyacentes a regiones que poseen proyección de desarrollo, inversión e infraestructura.

En los 70s una parte importante de la población urbana vivía en las únicas tres localidades de más de un millón de

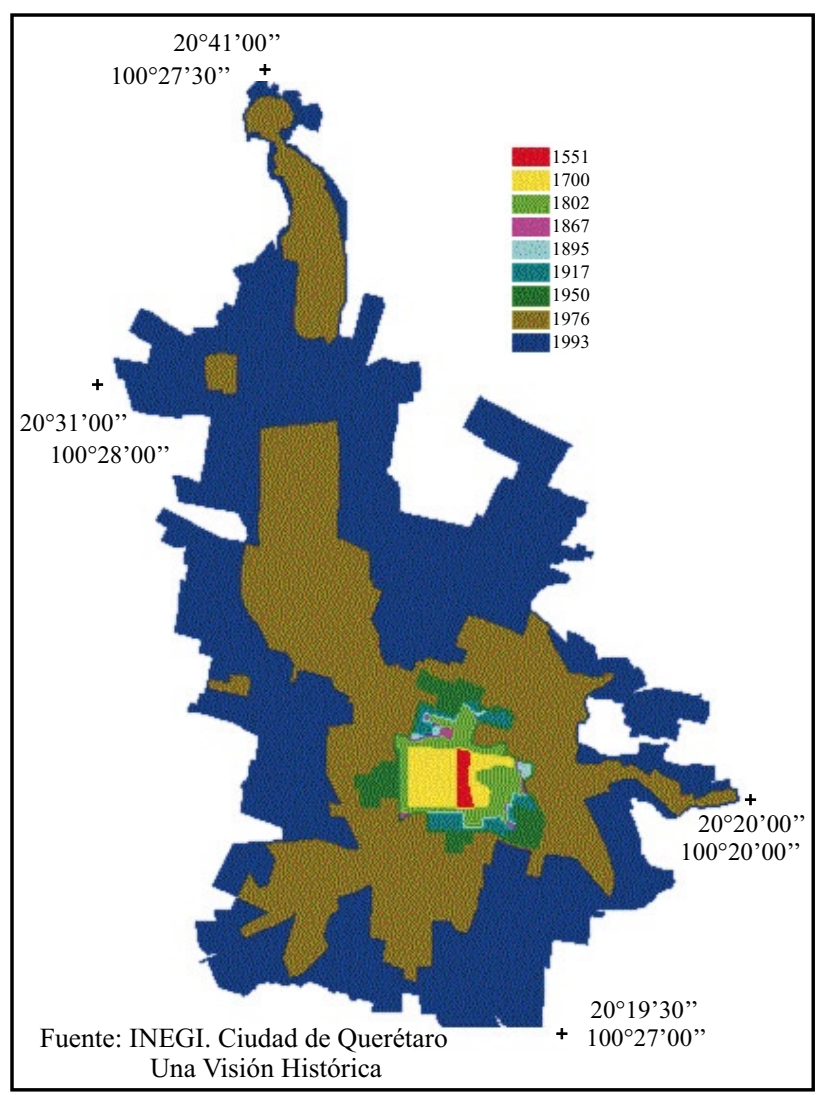

Figura 3. Mancha urbana de la ciudad de Querétaro 1950-1993. habitantes; para el 2000 aumentaron a nueve el número de localidades de ese tamaño. De las nueve ciudades millonarias, cinco se localizan en el centro del país: las zonas metropolitanas de México, Guadalajara, Puebla, León y Toluca; cuatro en el norte: La ZM de Monterrey, Tijuana, Ciudad Juárez y la ZM de Torreón Tabla 1.

El crecimiento físico de la ciudad de Querétaro, ha permitido el desbordamiento de la mancha urbana sobre los tres municipios vecinos, ha derivado en diversos problemas de contaminación y suministro de agua, entre otros. Estas características no están relacionadas solamente con el crecimiento, sino también con la falta de planeación oportuna, conformándose zonas sociales y económicamente segregadas dentro de la ZMCQ. Esta aglomeración es catalogada como una ciudad media por su población. En el 2000, el XX Censo General de Población contó 816,481 hababitantes.

Se han aplicado proyecciones en ciudades catalogadas en el rango de muy grandes (dentro de estas la Ciudad de Querétaro) y el rápido crecimiento demográfico que han presentado, hizo suponer que para el 2010 o 2020 cuatro de ellas llegarían a la categoría de millonarias: la zona metropolitana de Querétaro, Mérida, San Luís Potosí y Aguascalientes. Se debe tomar en cuenta que las tendencias se pueden alterar por al aplicar planes de desarrollo en estas ciudades. La mayor posibilidad de alcanzar esa cifra de habitantes la tiene las ZM de Querétaro y la de Mérida, ya que con cualquiera de las tres alternativas de aumento aplicadas: baja, media o alta, podrían alcanzar la cifra señalada Tabla 2 y Figura 4.

Particularmente la ZMCQ pasó de 221,852 a 816,481 habitantes de 1970 al 2000. La ZMCQ, ha concentrado prácticamente la mitad de la población estatal $(44.7 \%$ en 1995) y, a pesar del acelerado crecimiento de la población de los municipios de reciente incorporación, Querétaro ha crecido aún más rápido y pasó de concentrar 73.5 por ciento de la población metropolitana en 1970 a 78.5 por ciento, al cambio de siglo. De continuar las tendencias de crecimiento observadas, la concentración en el municipio de Querétaro llegará a 80 por ciento en 2020. Al cambio de siglo, la mancha urbana de la ZMCQ abarcaba ya partes importantes de cada uno de los cuatro municipios, aunque en menor medida del municipio de Huimilpan, que es el de más reciente incorporación con la ZMCQ.

En cuanto a la densidad de población promedio existen variaciones sustanciales, oscila entre 917 hasta 75 habitantes $/ \mathrm{km} 2$. En la Ciudad de Querétaro se observa la densidad especialmente alta que, en parte, refleja la presencia de asentamientos urbanos consolidados, mientras que Huimilpan muestra una densidad de población sensiblemente más baja, que guarda proporción con las zonas rurales y semi rurales.

Los indicadores sociodemográficos del estado muestran diversidad de procesos que orientan el fenómeno migratorio, en términos generales la dinámica actual, responde a relaciones económicas y vías de comunicación, en este 
Tabla 1. Población de las ciudades millonarias de México. Fuente: Gutierréz-MacGregor y González, 2004.

\begin{tabular}{|c|c|c|c|c|c|c|c|c|}
\hline Ciudades principales: & $\begin{array}{l}1970 \\
\text { Población }\end{array}$ & $\%$ & $\begin{array}{r}1980 \\
\text { Población }\end{array}$ & $\%$ & $\begin{array}{r}1990 \\
\text { Población }\end{array}$ & $\%$ & $\begin{array}{r}2000 \\
\text { Población }\end{array}$ & $\%$ \\
\hline ZM de la Ciudad de México & 8623156 & 36.2 & 43856923 & 35.2 & 15049636 & 29.2 & 17682176 & 27.1 \\
\hline ZM de Guadalajara & 1480472 & 6.2 & 2264602 & 5.7 & 2891333 & 5.6 & 3542120 & 5.4 \\
\hline ZM de Monterrey & 1213479 & 5.1 & 2001502 & 5.1 & 2560363 & 4.9 & 3214492 & 4.9 \\
\hline ZM de Puebla & 629344 & 2.6 & 1136875 & 2.9 & 1640695 & 3.2 & 2095601 & 3.2 \\
\hline ZM de León & 470209 & 2.0 & 722384 & 1.8 & 951521 & 1.8 & 1235081 & 1.9 \\
\hline Ciudad Juárez & 407370 & 1.7 & 544496 & 1.4 & 789522 & 1.5 & 1187275 & 1.8 \\
\hline ZM de Toluca & 114079 & 0.5 & 597350 & 1.5 & 821464 & 1.6 & 1151651 & 1.8 \\
\hline Tijuana & 327400 & 1.4 & 429500 & 1.1 & 698752 & 1.3 & 1148681 & 1.8 \\
\hline ZM de Torreón & 438461 & 1.8 & 689195 & 1.7 & 878289 & 1.7 & 1007291 & 1.5 \\
\hline Total de Población Urbana & 23770210 & 100.0 & 39305983 & 100.0 & 51696490 & 100.0 & 65310988 & 100.0 \\
\hline
\end{tabular}

sentido la influencia más importante la ejerce la autopista México-Querétaro, con la inclusión y el crecimiento de las actividades industriales y de servicios que en ella se han ido generando.

\section{El agua, recurso estratégico}

El agua es uno de los grandes recursos que ofrece la naturaleza y al mismo tiempo es una de las riquezas indispensables para la vida del hombre, es también un factor determinante en la distribución y desarrollo de diversas actividades económicas, de suerte, que cuando se quiere caracterizar una región por sus factores geoeconómicos este recurso natural es uno de los mas primordiales (Granados, 1996).

Dentro de la clasificación de recursos naturales le corresponde al agua estar catalogada dentro de los recursos renovables, no olvidando que el mal uso que se haga de ella, puede dar lugar a la disminución de ésta en ciertas regiones, aunque no cambia el balance de reservas de toda la Tierra.

En México son importantes las fuentes de agua subterránea y, en algunas regiones, son la única forma de abastecimiento de agua. Uno de los problemas más importantes del manejo de aguas subterráneas es la sobreexplotación de los acuíferos.

El Valle de Querétaro con un área aproximada de 42500 ha, se ubica al suroeste del Estado del mismo nombre. Su acuífero cubre los municipios de Querétaro, Corregidora y parte del Marqués. De acuerdo con el padrón levantado por la CNA (1998), había 239 pozos en operación (GutiérrezCarrillo, et al., 2002).

De 1970 a 1986 se registraron abatimientos de 5 a más de $35 \mathrm{~m}$, lo que significó una explotación, equivalente de 0.3 hasta poco más de $2 \mathrm{~m}$ por año. Actualmente las profundidades del nivel van de los 50 a los $70 \mathrm{~m}$ en el valle, con los niveles más profundos bajo la mancha urbana y el parque industrial, a causa de la gran concentración local de pozos, y de 70 a más de $110 \mathrm{~m}$ en las partes altas debido a la pendiente topográfica. Los caudales comunes de extracción, de 30 a 60 lps.

De continuar la tendencia actual, aunada a las demandas que existirán por los nuevos asentamientos surgidos a lo largo de las nuevas carreteras, en el 2010, el abatimiento promedio de los niveles estáticos en el Valle de Querétaro alcanzará los $8 \mathrm{~m}$ año-1 y la profundidad promedio al nivel estático será del orden de $175 \mathrm{~m}$.

Debido a las características climáticas propias de la zona, semiseca y la mayor parte del agua que escurre no se puede aprovechar, hacen necesario que exista un aprovechamiento óptimo del recurso.

Hace aproximadamente tres décadas que las extracciones de agua en los acuíferos, en especial en el acuífero del Valle de Querétaro empezaron a sobrepasar su recarga natural. La disponibilidad de agua proveniente de estos acuíferos en el mediano plazo tiene un matiz de incertidumbre, por el hecho de que se desconoce la cantidad de agua que queda aún almacenada.

La demanda de agua para usos industriales y domésticos

Tabla 2. Tendencias demográficas de cuatro ciudades seleccionadas para los años 2010 y 2020. Fuente: Gutierréz-MacGregor y González, 2004.

\begin{tabular}{|c|c|c|c|c|c|c|}
\hline \multirow[t]{3}{*}{ Ciudad } & \multicolumn{3}{|c|}{ Alternativas en el 2010} & \multicolumn{3}{|c|}{ Alternativas en el 2020} \\
\hline & Baja & Media & Alta & Baja & Media & Alta \\
\hline & \multicolumn{3}{|c|}{ Habitantes (en miles) } & \multicolumn{3}{|c|}{ Habitantes (en miles) } \\
\hline Querétaro & 983 & 964 & 998 & 1068 & 1126 & 1159 \\
\hline Mérida & 949 & 960 & 980 & 1005 & 1054 & 1090 \\
\hline San Luis Potosí & 927 & 939 & 960 & 980 & 1008 & 1049 \\
\hline Aguascalientes & 827 & 854 & 870 & 927 & 972 & 1000 \\
\hline
\end{tabular}




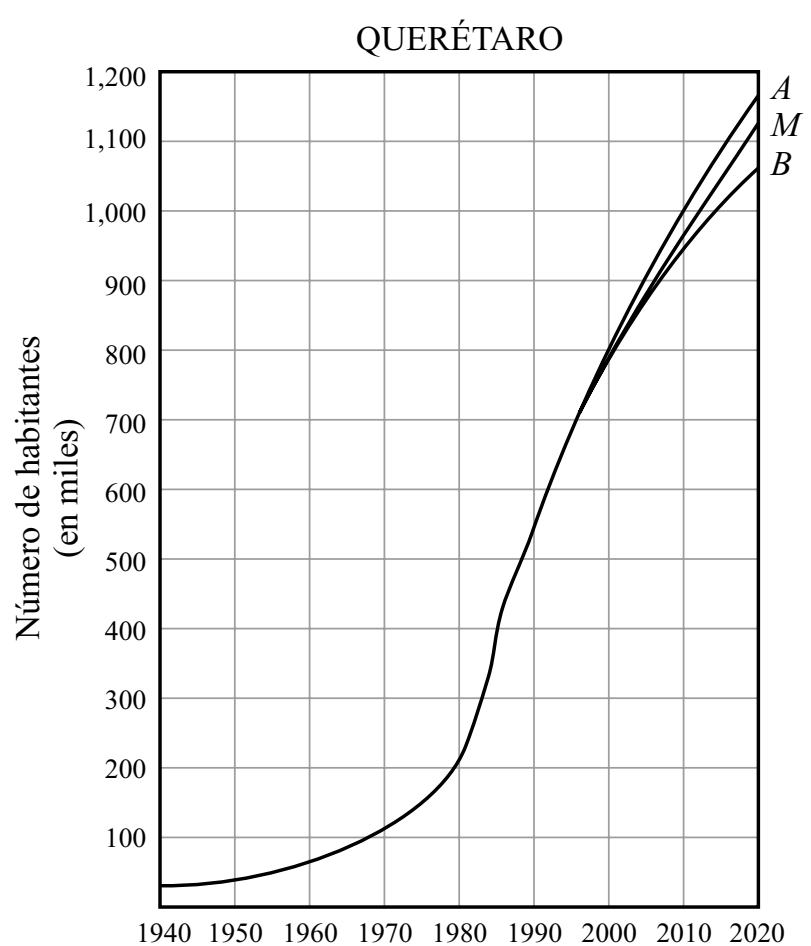

Figura 4. Tendencia demográfica 1940 - 2020

ha crecido en las últimas décadas. La falta de agua alteraría el ecosistema del que forma parte. (Salazar, 2004). El uso indiscriminado puede comprometer nuestro futuro. El agua del siglo XXI puede ser un bien estratégico, si es que no en la actualidad ya es motivo de conflicto entre países.

\section{Conclusiones}

En la evolución de los espacios metropolitanos (y su consecuente demanda de agua), la dinámica de la organización funcional, la accesibilidad y la estructura económica y espacial del espacio metropolitano, ha estado vinculada con la implementación de los megaproyectos de transporte.

Las grandes obras de infraestructura, como las redes carreteras; traerán consigo crecimientos urbanos formales e informales, con sus consecuentes demandas de servicios. Las estimaciones sobre el crecimiento futuro de la poblaciones en la ZMCQ son importante para que las autoridades respectivas, cuenten con algunas bases, para diseñar y establecer su política demográfica y demandas de servicios.

De persistir la actual explotación de aguas subterráneas con sus respectivos aumentos por las demandas de los nuevos asentamientos, podría generar severas limitaciones en el uso futuro del recurso. Específicamente, podría dejar de ser rentable algunas actividades económicas debido a que los niveles de bombeo seran más profundos y costosos.

\section{Referencias bibliográficas}

Aguilar, G. Coordinador, 2006, Las grandes aglomeraciones y su periferia regional experiencias en Latinoamérica y España. Porrua. México.

Ampe, F., 1995, Technopole development in Euralille. In Transport and urban development, Ed E \& FN Spon, London.

Banister, D., 1995, Transport and urban development, Ed E \& FN Spon, London.

Granados, R., 1996, Distribución Uso y Aprovechamiento del Recurso Agua en el estado de Querétaro". Colegio de Geografía, Facultad de Filosofía y Letras, UNAM, México, Tesis Licenciatura 176 p.

Gutiérrez-Carrillo N; Palacios-Vélez E; Peña-Díaz S y Palacios-Vélez O., 2002, Escenarios para el aprovechamiento sustentable del acuífero del Valle de Querétaro. Agrociencia volumen 36, número 1, enero-febrero, Pág. 1-10.

Gutiérrez-McGregor Ma. T. y González S., 2004. Dinámica y Distribución espacial de la población urbana en México 1970-2000, Instituto de Geografía, UNAM, 158. p.

H. Ayuntamiento de Querétaro, 1997, Plan de Desarrollo Integral del Municipio de Querétaro 1997-2000, Edit. Qro., México.

Hanson, S. 1986. The geography of urban transportation, Guillford, New York.

Instituto Nacional de Estadística, Geografía e Informática (INEGI), 2002, Una visión Histórica, México.

Salazar G. J. A., 2004, El agua, un bien para todos. Conservación recuperación y usos. Ediciones Universidad de Salamanca, España. pp. 117-133.

Secretaria de Comunicaciones y Transporte (SCT), 2001a, Plan Puebla Panamá, Proyectos Estratégicos. Secretaría de Comunicaciones y Transporte, México.

Secretaria de Comunicaciones y Transporte (SCT), 2001b, Programa sectorial de comunicación y transporte 2001-2006. El sector que nos integra y moderniza. $303 \mathrm{p}$.

Secretaria de Comunicaciones y Transporte (SCT), Enfoque Regional y Sustentabilidad. Enfoque regional.http://www.sct.gob.mx. Consultada el 10 de agosto de 2004.

Villaseñor, A., 2004, Los Megaproyectos del sector transporte y su incidencia en la Zona Metropolitana de la Ciudad de México. México, Universidad Nacional Autónoma de México, Tesis doctoral. 205 p.

Manuscrito aceptado: Enero 6, 2006

Manuscrito corregido recibido: Octubre 30, 2007

Manuscrito aceptado: Diciembre 2, 2007 